\title{
Experience- and Sex-Dependent Intrinsic Plasticity in the Zebra Finch Auditory Cortex during Song Memorization
}

\author{
Andrew N. Chen ${ }^{1}$ and ${ }^{\circ}$ C. Daniel Meliza ${ }^{1,2}$ \\ ${ }^{1}$ Neuroscience Graduate Program, and ${ }^{2}$ Psychology Department, University of Virginia, Charlottesville, Virginia 22904
}

For vocal communicators like humans and songbirds, survival and reproduction depend on highly developed auditory processing systems that can detect and differentiate nuanced differences in vocalizations, even amid noisy environments. Early auditory experience is critical to the development of these systems. In zebra finches and other songbirds, there is a sensitive period when young birds memorize a song that will serve as a model for their own vocal production. In addition to learning a specific tutor's song, the auditory system may also undergo critical developmental processes that support auditory perception of vocalizations more generally. Here, we investigate changes in intrinsic spiking dynamics among neurons in the caudal mesopallium, a cortical-level auditory area implicated in discriminating and learning species-specific vocalizations. A subset of neurons in this area only fire transiently at the onset of current injections (i.e., phasic firing), a dynamical property that can enhance the reliability and selectivity of neural responses to complex acoustic stimuli. At the beginning of the sensitive period, just after zebra finches have fledged from the nest, there is an increase in the proportion of caudal mesopallium neurons with phasic excitability, and in the proportion of neurons expressing Kv1.1, a low-threshold channel that facilitates phasic firing. This plasticity requires exposure to a complex, noisy environment and is greater in males, the only sex that sings in this species. This shift to more phasic dynamics is therefore an experience-dependent adaptation that could facilitate auditory processing in noisy, acoustically complex conditions during a key stage of vocal development.

Key words: auditory learning; developmental plasticity; firing dynamics; intrinsic plasticity; vocal development; zebra finch

Significance Statement

Auditory experience early in life shapes how humans and songbirds perceive the vocal communication sounds produced by their species. However, the changes that occur in the brain as this learning takes place are poorly understood. In this study, we show that in young zebra finches that are just beginning to learn the structure of their species' song, neurons in a key cortical area adapt their intrinsic firing patterns in response to the acoustic environment. In the complex, cocktail-party-like environment of a colony, more neurons adopt transient firing dynamics, which can facilitate neural coding of songs amid such challenging conditions.

\section{Introduction}

Vocal communicators require auditory systems that can reliably decode the rich acoustic structure of vocalizations (Singh and Theunissen, 2003; Wang et al., 2008). In humans, songbirds, and other species that learn how to vocalize through imitation, early experience hearing conspecific vocalizations influences the development of both auditory perception and vocal production (Doupe and Kuhl, 1999). A classic example is the perceptual nar-

\footnotetext{
Received Sept. 3, 2019; revised Dec. 9, 2019; accepted Dec. 23, 2019.

Author contributions: A.N.C. and C.D.M. designed research; A.N.C. and C.D.M. performed research; A.N.C. and C.D.M. analyzed data; A.N.C. and C.D.M. wrote the paper.

This work was supported in part by the Thomas F. and Kate Miller Jeffress Memorial Trust and by the University of Virginia Brain Institute. We thank M. Bjoring for assistance analyzing acoustic recordings; F. Ahsan and K. Hess for help with analyzing immunofluorescence images; and $\mathrm{G}$. Edgerton for feedback on the paper.

The authors declare no competing financial interests.

Correspondence should be addressed to C. Daniel Meliza at cdm8j@virginia.edu.

https://doi.org/10.1523/JNEUROSCI.2137-19.2019

Copyright $\odot 2020$ the authors
}

rowing that occurs in humans during the first year of life, in which exposure to speech establishes the phonetic distinctions an infant is able to perceive (Werker and Lalonde, 1988; Kuhl, 2004). Infants are exquisitely sensitive to the statistics of speech sounds (Maye et al., 2002), but the neural plasticity underlying this important form of learning is poorly understood.

As in humans, early auditory experience is critical to vocal communication in zebra finches (Taeniopygia guttata). Around $25 \mathrm{~d}$ of age, juvenile males begin to memorize the song of an adult male tutor whom they will learn to imitate (Gobes et al., 2019). Birds who do not hear song during this sensory acquisition period are unable to produce species-typical songs as adults, even if they are exposed to a tutor later in life (Marler and Tamura, 1964; Eales, 1985). Although the failure to develop normal song undoubtedly reflects the lack of a specific model to guide sensorimotor learning, it is also important to consider how the early acoustic environment impacts auditory processing more generally (Woolley, 2012; Moore and Woolley, 2019). In rodents, the 
statistics of sensory experience during an early critical period have lasting effects on cortical tuning (Zhang et al., 2001; de Villers-Sidani et al., 2007; Levelt and Hübener, 2012). Similarly, songbirds raised in acoustically impoverished environments exhibit a range of behavioral and physiological deficits consistent with abnormal auditory processing (Sturdy et al., 2001; Amin et al., 2013; Chen et al., 2017). These auditory deficits could contribute significantly to the inability of isolated birds to learn how to sing normally, for instance by causing auditory feedback to be distorted, less precise, or harder to perceive in a background of conspecific vocalizations.

To test the hypothesis that experience-dependent development of auditory processing can occur in songbirds independently of song memorization, we measured how the physiological properties of auditory neurons are affected by early experience, using rearing conditions that separately control exposure to a tutor song and exposure to the complex acoustic background of songs and calls juveniles hear in normal colonial breeding conditions. We focused on the caudal mesopallium (CM), a corticallevel auditory area (Wang et al., 2010) that responds selectively to familiar conspecific songs (Gentner and Margoliash, 2003; Meliza and Margoliash, 2012) and could be a site of plasticity during development. We previously found that in juvenile finches, the putatively excitatory neurons in $\mathrm{CM}$ have diverse spiking dynamics (Chen and Meliza, 2018). Some neurons produce sustained responses to depolarizing step currents, whereas others respond only transiently at the beginning of the stimulus (i.e., phasic excitability). These firing patterns persist when fast synaptic transmission is blocked, indicating that they depend on intrinsic membrane properties. Specifically, like phasic cells in the auditory hindbrain (Bal and Oertel, 2001), phasic CM neurons express a low-threshold potassium current that activates close to the spike threshold and suppresses repetitive firing. When phasic neurons are stimulated with broadband input currents, the low-threshold potassium current improves encoding of the temporal modulation frequencies found in zebra finch song (Chen and Meliza, 2018) and, in simulations, the responses of phasic neurons to song are less sensitive to variation in spontaneous activity (Bjoring and Meliza, 2019). Thus, intrinsic dynamics are likely to strongly influence auditory processing and could be a target of experience-dependent plasticity.

\section{Materials and Methods}

Animals. All procedures were performed according to NIH guidelines and protocols approved by the University of Virginia IACUC. Zebra finches (Taeniopygia guttata) were bred from our local colony. All birds received finch seed (Abba Products) and water ad libitum and were kept on a 16:8 h light/dark schedule in temperature- and humidity-controlled rooms at $22-24^{\circ} \mathrm{C}$.

Sex was determined from plumage coloration in older animals or by PCR amplification of the CHD-1 gene, which has different lengths on the $\mathrm{Z}$ and W sex chromosomes. Genomic DNA was isolated from feathers (Qiagen DNAeasy Blood and Tissue Kit) or from blood stored in a 5\% Chelex-100 solution. The forward primer sequence was YTKCCAA GRATGAGAAACTG, and the reverse primer sequence was TCTGCAT CACTAAAKCCTTT. Because females are heterogametic, these primers yield two products $\sim 350$ and $400 \mathrm{bp}$ in length in females, whereas male DNA only yields the shorter product. No-template and positive male and female controls were included in each PCR batch. Note that any crosssample contamination would result in males being misidentified as female, which would reduce the size of the sex difference observed here rather than producing a false-positive.

Experimental rearing conditions. All of the animals in the study were bred in individual cages that were initially placed in a room housing dozens of male and female finches of varying ages. Colony-reared (CR) chicks remained in the colony room until they were used in an experiment. Juveniles were separated from their parents after $\sim 35 \mathrm{~d}$ and housed with siblings or in large aviaries. In the female-reared (FR) condition, the cage was moved into an acoustic isolation box (Eckel Industries) after the first egg hatched. Within $5 \mathrm{~d}$, the father was removed from the cage and housed elsewhere. In some cases, an unrelated female was added to assist with provisioning. Male and female FR chicks were separated after they began to show plumage differences $(\sim 40 \mathrm{~d})$ so that females would not be exposed to FR males' attempts to sing. Pair-reared (PR) families were also moved to acoustic isolation boxes after the first chick hatched, but the father was not removed. Visually isolated (VI) chicks remained in the colony room, but in cages that were separated from each other by opaque barriers. As with the FR condition, the father was removed within $5 \mathrm{~d}$ of the first egg hatching. Visual isolates could therefore hear the songs of adult males and call to them but could not socially interact. Some subjects may have been able to see males in a large flight aviary $>1 \mathrm{~m}$ away.

Acoustic recordings of ambient noise were obtained for one FR and one VI clutch. A small hole was drilled in the lid of the nest box, a piece of foam rubber padding with a cavity for a microphone was glued above the hole, and an omnidirectional lavalier microphone (Shure SM93) was placed in the cavity. The signal was amplified and digitized at $44.1 \mathrm{kHz}$ using a Focusrite Scarlett $2 \mathrm{i} 2$ and recorded directly to disk using custom software (jrecord v2.1.4; https://github.com/melizalab/jill). Before installing the lid on the sound box, a recording was made of a $94 \mathrm{~dB}$ SPL calibration signal emitted by an R8090 (Reed Instruments) sound level calibrator placed immediately above the hole on the nest side of the lid. The recording gain was kept constant throughout the recording. Recordings were obtained over $3 \mathrm{~d}$ in each condition while chicks were $\sim 1$ week old.

Brain slice preparation and electrophysiology. Whole-cell recordings were obtained from 575 broad-spiking neurons in acute brain slices from 168 zebra finches, using methods adapted from (Zhao et al., 2011). These data included 169 neurons analyzed in a previous publication (Chen and Meliza, 2018) that focused only on juvenile birds (P22-P40). Birds were administered a lethal intramuscular injection of Euthasol (pentobarbital sodium and phenytoin sodium; $200 \mathrm{mg} / \mathrm{kg}$; Hospira) and perfused transcardially with ice-cold cutting buffer (in mM: $92 \mathrm{NaCl}, 2.5 \mathrm{KCl}, 1.2$ $\mathrm{NaH}_{2} \mathrm{PO}_{4}, 30 \mathrm{NaHCO}_{3}, 20$ HEPES, 25 glucose, 5 sodium ascorbate, 2 thiourea, 3 sodium pyruvate, $10 \mathrm{MgSO}_{4} \cdot 7 \mathrm{H}_{2} \mathrm{O}, 0.5 \mathrm{CaCl}_{2} \cdot 2 \mathrm{H}_{2} \mathrm{O}, \mathrm{pH}$ $7.3-7.4,300-310 \mathrm{mmol} / \mathrm{kg}$ ). In older animals, the cutting buffer contained $93 \mathrm{~mm} \mathrm{~N}$-methyl-D-glucamine instead of $\mathrm{NaCl}$. The brain was blocked using a custom 3D printed brain holder (http://3dprint.nih.gov/ discover/3dpx-003953). Three hundred micrometer sections were cut in room-temperature cutting buffer on a VF-200 Compresstome (Precisionary Instruments), and then transferred to $32^{\circ} \mathrm{C}$ cutting buffer for 10-15 min for initial recovery. Sections were then transferred to roomtemperature holding buffer (in mM: $92 \mathrm{NaCl}, 2.5 \mathrm{KCl}, 1.2 \mathrm{NaH}_{2} \mathrm{PO}_{4}, 30$ $\mathrm{NaHCO}_{3}, 20$ HEPES, 25 glucose, 5 sodium ascorbate, 2 thiourea, 3 sodium pyruvate, $2 \mathrm{MgSO}_{4} \cdot 7 \mathrm{H}_{2} \mathrm{O}, 2 \mathrm{CaCl}_{2} \cdot 2 \mathrm{H}_{2} \mathrm{O}$, pH 7.3-7.4, 300-310 $\mathrm{mmol} / \mathrm{kg}$ ) to recover for at least $1 \mathrm{~h}$ before use in recordings. All solutions were bubbled continuously with $95 \% \mathrm{O}_{2} \cdot 5 \% \mathrm{CO}_{2}$ mixture starting at least $10 \mathrm{~min}$ before use.

Slice recordings were conducted in a RC-26G recording chamber (Warner Instruments) perfused with standard recording ACSF (in mM: $124 \mathrm{NaCl}, 2.5 \mathrm{KCl}, 1.2 \mathrm{NaH}_{2} \mathrm{PO}_{4}, 24 \mathrm{NaHCO}_{3}, 5$ HEPES, 12.5 glucose, 2 $\mathrm{MgSO}_{4} \cdot 7 \mathrm{H}_{2} \mathrm{O}, 2 \mathrm{CaCl}_{2} \cdot 2 \mathrm{H}_{2} \mathrm{O}$; $\mathrm{pH} 7.3-7.4,300-310 \mathrm{mmol} / \mathrm{kg}$ ) at a rate of $1-2 \mathrm{ml} / \mathrm{min}$ at $32^{\circ} \mathrm{C}$, which was the highest and closest to physiological temperatures at which slices remained healthy and recordings stable. Whole-cell patch-clamp recordings were obtained under $60 \times$ infrared $(900 \mathrm{~nm})$ DIC optics. CM was located relative to the lamina mesopallialis ventralis (LMV) and the internal occipital capsule (CIO), which both comprise dense myelinated fibers visible as dark bands under bright-field or IR illumination. Electrode placement was confirmed after recording by taking multiple overlapping images of the section at $4 \times$ with the electrode in place, and then stitching the images together using the Grid/ Collection plugin from ImageJ v2.0.0-rc-69. Of the 323 neurons that could be localized in this way, most $(78 \%)$ were in the lateral subdivision of CM (CLM), which is superior to CIO in this sectioning plane; $12 \%$ 
were considered to be in medial CM (CMM), which lies above a region of the nidopallium where myelin is lighter and more diffuse (presumably L2b); and 10\% were on the border of CLM and CMM and could not be unambiguously assigned to either area. The distribution of recording sites did not differ significantly between ages $\left(\chi_{(10)}^{2}=16.8, p=0.08\right)$ or rearing conditions $\left(\chi_{(6)}^{2}=6.1, p=0.42\right)$. Recording pipettes were pulled from filamented borosilicate glass pipettes ( $1.5 \mathrm{~mm}$ outer diameter, 1.10 $\mathrm{mm}$ inner diameter; Sutter Instruments) using a P-1000 Micropipette Puller (Sutter Instruments) and were filled with internal solution (in mM: 135 K-gluconate, 10 HEPES, $8 \mathrm{NaCl}, 0.1$ EGTA, 4 MgATP, 0.3 NaGTP, $10 \mathrm{Na}$-phosphocreatine, pH 7.2-7.3, 290-300 mmol/kg). For $\sim$ one-half of our recordings, we prepared solutions to match the elevated osmolarity reported in zebra finch CSF (Bottjer, 2005). ACSF solutions were adjusted to $345-355 \mathrm{mmol} / \mathrm{kg}$, and internal solutions to $335-345 \mathrm{mmol} /$ $\mathrm{kg}$. However, no noticeable differences in slice health or firing patterns were observed with this modification.

Electrodes had a resistance of 3-8 $\mathrm{M} \Omega$ in the bath. Voltages were measured with a MultiClamp 700B amplifier (Molecular Devices) in current-clamp mode, low-pass filtered at $10 \mathrm{kHz}$, and digitized at $40 \mathrm{kHz}$ with a Digidata 1440A. Pipette capacitance was neutralized, and 8-12 $\mathrm{M} \Omega$ of series resistance was subtracted by bridge balance. Recorded voltage was corrected offline for measured liquid junction potential of 11.6 $\mathrm{mV}$ at $32^{\circ} \mathrm{C}$. Current injections and data collection were controlled by pClamp v10.4 (Molecular Devices). Neurons were excluded from further analysis if the resting membrane potential was $>-55 \mathrm{mV}$ or if action potentials failed to cross $-10 \mathrm{mV}$. Two hyperpolarizing current injections at different amplitudes $(500-1000 \mathrm{~ms})$ were used to monitor input and series resistance. Resting input resistance $\left(R_{\mathrm{m}}\right)$, and capacitance $\left(C_{\mathrm{m}}\right)$ were estimated from the responses to these step currents by fitting a sum of two exponential functions to the voltage decay (using Chebyshev polynomial regression). Recording sweeps were excluded if the input resistance, series resistance, or resting potential deviated by $>20 \%$ from baseline.

Each neuron was stimulated with depolarizing step currents $2 \mathrm{~s}$ in duration at a range of amplitudes. Analysis of the responses followed the same procedures described previously (Chen and Meliza, 2018). Action potentials were detected as positive-going crossings of a threshold $35 \%$ of the difference between the peak of the first spike and its takeoff point. Only spikes that peaked at least $10 \mathrm{mV}$ above the detection threshold and that were $<8 \mathrm{~ms}$ in width (at half-height) were included. This procedure excluded the broad voltage oscillations observed in some neurons during prolonged depolarization. Cells were classified as narrow-spiking if the spike width was $<0.8 \mathrm{~ms}$ or if maximum steady-state spike rate was $>30$ $\mathrm{Hz}$, and were excluded from analysis.

The response duration in each sweep was measured as the time elapsed between the first and the last spikes of the pulse. In responses with only one spike, the duration $(\Delta t)$ was defined as the width of the spike plus the duration of the afterhyperpolarization. The emphasis of this study was on phasic firing caused by a low-threshold potassium current, which produces outward rectification at $\sim-60 \mathrm{mV}$ (Chen and Meliza, 2018). We therefore excluded sweeps in which depolarization block occurred, as evidenced by a steady-state potential $>-45 \mathrm{mV}$. However, sweeps with high steady-state potentials were not excluded from five neurons that had unusually high rheobases and had to be depolarized $>-45 \mathrm{mV}$ to spike at all. Cells were classified as phasic if they did not fire during the last 500 $\mathrm{ms}$ of the step current in response to any magnitude current; otherwise, they were classified as tonic. Note that in contrast to our previous study (Chen and Meliza, 2018), we no longer distinguish an "intermediatespiking" class, because the immunofluorescence data suggest that Kv1.1, which is likely to be the underlying low-threshold potassium channel that drives phasic firing, is distributed continuously across the population.

Immunofluorescence. Animals were administered a lethal intramuscular injection of Euthasol and perfused transcardially with a $10 \mathrm{U} / \mathrm{ml}$ solution of sodium heparin in PBS (in mm: $10 \mathrm{Na}_{2} \mathrm{HPO}_{4}, 154 \mathrm{NaCl}, \mathrm{pH}$ 7.4) followed by $4 \%$ formaldehyde (in PBS). Brains were immediately removed from the skull, postfixed overnight in $4 \%$ formaldehyde at $4^{\circ} \mathrm{C}$, cryoprotected in $30 \%$ sucrose (in $100 \mathrm{~mm} \mathrm{Na} \mathrm{HPO}_{4}, \mathrm{pH} 7.4$ ), blocked saggitally into hemispheres, embedded in OCT, and stored at $-80^{\circ} \mathrm{C}$. Sections were cut from one hemisphere at $40 \mu \mathrm{m}$ on a cryostat, rinsed four times in PBS, and then incubated in citrate buffer $(0.01 \mathrm{M}, \mathrm{pH} 8.5)$ at $80^{\circ} \mathrm{C}$ for $25 \mathrm{~min}$. The sections were cooled to room temperature, washed twice in PBS, permeabilized in PBS-T ( $0.1 \%$ Tween in PBS), and blocked in $5 \%$ goat serum and $2 \%$ glycine in PBS-T. The tissue was stained for Kv1.1 using a monoclonal mouse antibody (1:500 in blocking solution; UC Davis/NIH Neuromab clone K20/78; RRID:AB_10673165) for $60-72 \mathrm{~h}$ at $4^{\circ} \mathrm{C}$. Sections were washed four times for $15 \mathrm{~min}$ in PBS-T and then incubated with a fluorescent secondary antibody (1:2000 in PBS-T; goat anti-mouse IgG1 conjugated to AlexaFluor 488; Invitrogen; RRID: AB_2534069). The tissue was washed twice with PBS, counterstained for Nissl (NeuroTrace 640/660, 1:1000 in PBS; ThermoFisher Scientific, catalog \#N21483; RRID:AB_2572212), washed four times with PBS, and then mounted and coverslipped in Prolong Gold with DAPI (ThermoFisher Scientific, catalog \#P36934; RRID:SCR_015961).

Stained tissue was imaged with a $40 \times$ objective (water-immersion, NA 1.2) on a Zeiss LSM 800 confocal microscope in stacks with $0.44 \mu \mathrm{m}$ between optical sections. Higher-power images for illustration were obtained with a $63 \times$ objective (water-immersion, NA 1.2). At least three stacks were imaged in each section in locations that spanned the dorsoventral and caudorostral axes. Images were collected from at least two sections in each animal, preferably one each from the medial and lateral subdivisions of CM. Left and right hemispheres were balanced across groups. To quantify expression, we counted the number of Kv1.1positive neurons in each stack manually in Imaris v9.2.1 after using the automatic background subtraction, with the default smoothing radius of $39.9 \mu \mathrm{m}$. The total number of neurons in each stack was counted from the Nissl channel (in a few cases, we used DAPI when the NeuroTrace staining was poor) using the automatic spot detection function in Imaris, followed by manual curation. Samples were processed and analyzed in three batches of 8-11 animals each, and each batch contained samples from every condition. Identical laser power and gain settings were used for the Kv1.1 channel in all the samples from each batch, but the Nissl and DAPI settings were adjusted freely to optimize signal quality. Experimenters were blind to animal identity and rearing condition throughout staining, image collection, and analysis, with the exception of one brain (CR, P19) that was repeated using the other hemisphere because none of the sections in the first batch contained CM.

The immunogen for the anti-Kv1.1 antibody was a synthetic peptide from the (intracellular) C-terminus of rat Kv1.1. The corresponding sequence in the zebra finch ortholog (KCNA1) is $74 \%$ identical, and a BLAST query against the zebra finch genome yielded no other hits. We validated the antibody by comparing staining patterns to in situ images from the ZEBrA gene expression brain atlas (ZEBrA Database, 2019). As in the atlas, cellular staining was much lower in L2a compared with the mesopallium and was absent in the habenula. In contrast, the two rabbit polyclonal antibodies we tried either failed to stain neurons in KCNA1positive regions or stained cell bodies in KCNA1-negative regions. Each staining batch included control sections that were incubated in blocking solution without primary antibody. Staining from the secondary antibody in these sections was present in the neuropil but essentially nonexistent in cell bodies.

Experimental design and statistical analyses. The objective of the study was to determine the degree to which the intrinsic spiking dynamics of neurons in the caudal mesopallium depend on age, sex, and experience. Ages were sampled between P18 (i.e., $18 \mathrm{~d}$ post-hatch) and P344, then binned into intervals corresponding to key developmental stages. Experience was manipulated through rearing conditions as described above. Some factors were not fully crossed; for example, the effects of PR and VI conditions were only tested between P30-P40. The effects of age, sex, and acoustic experience were measured using whole-cell electrophysiology and immunohistochemistry. The experimental design was not preregistered.

Statistical inference was performed using generalized linear mixedeffects models (GLMMs) with bird and family identity as random effects to account for repeated measures. Age group and rearing condition were included as fixed effects. Contrasts were planned with P18-P20 and FR as baselines.

For average response duration, individual sweeps were treated as the unit of observation. The error model was Gaussian, and neuron identity 
was added as a random effect. The variance of this effect was allowed to differ by age and condition (i.e., we estimated the between-neuron variance separately for each age/condition, which is consistent with the obvious heteroscedasticity of the data). The rationale for not pooling all the duration measurements within neurons is that individual responses tended to be either sustained or transient, and the duration did not depend much on the stimulus amplitude. GLMMs provide partial pooling, which made the estimates less sensitive to outlier trials in which spontaneous activity caused an isolated spike to occur near the end of the current step. The credible intervals in this sweep-level model were in better agreement with the raw data.

The proportion of phasic neurons was estimated using a more stringent approach in which neurons were only classified as phasic if they did not fire any action potentials during the last $500 \mathrm{~ms}$ of the current step. In this model, individual neurons were the unit of analysis and the dependent variable had a binomial error model.

The proportion of Kv1.1-positive neurons was also estimated with a binomial GLMM, with image stacks treated as the unit of observation. Sex, rearing condition, hemisphere, and mediolateral position were included as fixed effects. Section, bird, and family were included as random effects.

Because not every animal could be sexed and because dividing up the groups by sex reduced the effective sample size, the effect of sex on electrophysiological properties was analyzed separately in a model that only included CR animals. The electrophysiology rescue experiments were also analyzed in a separate model that only included animals between P30-P40. These experiments were performed before we had fully identified the narrow period between P30-P35 when phasic excitability is at its peak, so a broader range of ages was used.

Parameter estimates and credible intervals were obtained using MCMCglmm v3.5.2, which draws random samples from the joint posterior distribution of the model using Markov chain Monte Carlo sampling. Prior distributions were weakly informative: for fixed effects, normal with variance of 1000 and for variances, an inverse Wishart with variance 1 and degree of belief 0.002 . The number of samples and thinning were chosen to achieve adequate mixing (Gelman-Rubin diagnostic $\leq 1.01$ ) and an effective sample size of at least 1000 for every parameter-of-interest. Statistical tests were performed by determining the proportion of samples that exceeded the value for the null hypothesis; for example, the average response duration for P18-P20 FR birds was greater than the value for P65-P70 FR in 960/1000 samples, leading to a $p$ value of 0.04 .

\section{Results}

\section{Intrinsic dynamics are plastic throughout song learning}

We measured intrinsic excitability in zebra finches starting at age P18, when chicks fledge from the nest, through the sensory acquisition and sensorimotor learning periods, and into adulthood (Fig. 1A). We made whole-cell recordings from slices of CM (Fig. $1 B)$ and characterized the spiking patterns produced in response to step currents. As in our previous study, the putatively excitatory (broad-spiking) neurons exhibited diverse patterns of spiking responses, ranging from neurons that responded throughout

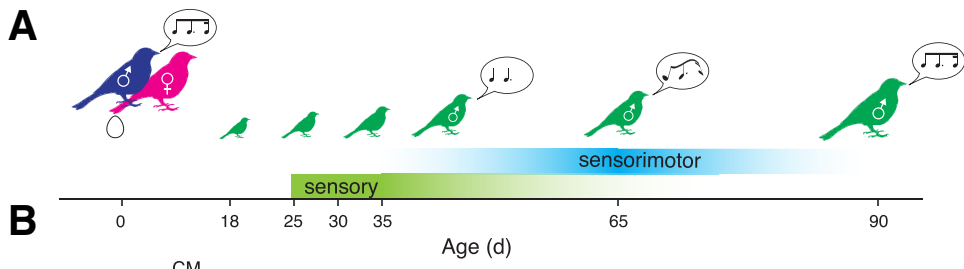

Figure 1. Intrinsic dynamics of CM neurons change over development. $\boldsymbol{A}$, Diagram of zebra finch auditory and vocal learning. $\boldsymbol{B}$, phasic spiking from a P37 bird of unknown sex. $\boldsymbol{E}$, Average response duration (log scale) as a function of age. Filled circles

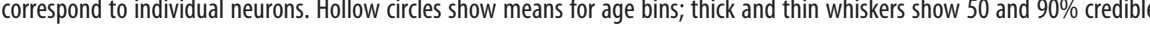
(GLMM; see Materials and Methods). Numerals above the plot indicate the number of birds in each group. with P18-P20, phasic neurons are more prevalent at P30-P35 $(p<0.001), P 36-P 40(p=0.018)$, and in adults $(p=0.006)$. Compared with P30-P35, phasic neurons are less prevalent at P36-P40 $(p=0.012)$ and P65-P70 $(p=0.020)$.

the stimulus (i.e., tonic excitability; Fig. 1C) to neurons that only fired at the onset (i.e., phasic excitability; Fig. 1D). We quantified phasic excitability using a continuous metric that was the logscaled response duration averaged across all current steps; decreases in response duration indicate a greater tendency for phasic firing. We also classified each neuron as tonic or phasic, depending on whether it responded in the last $500 \mathrm{~ms}$ of the stimulus for any current stimulus.

The distribution of intrinsic dynamics among CM neurons differed dramatically across development. In newly fledged birds (P18-P20), almost every neuron was tonic. Phasic excitability increased with age, reaching a peak between P30-P35 (Fig. $1 E, F)$. After this peak, phasic firing decreased as the sensory acquisition phase closed (P65-P70) and then increased again in adulthood $(\mathrm{P} 100+)$.

\section{Intrinsic plasticity during song memorization depends on sex and experience}

Because the peak in phasic excitability occurred at the height of sensory acquisition for song, we hypothesized that this intrinsic plasticity was involved in song memorization, either directly by 

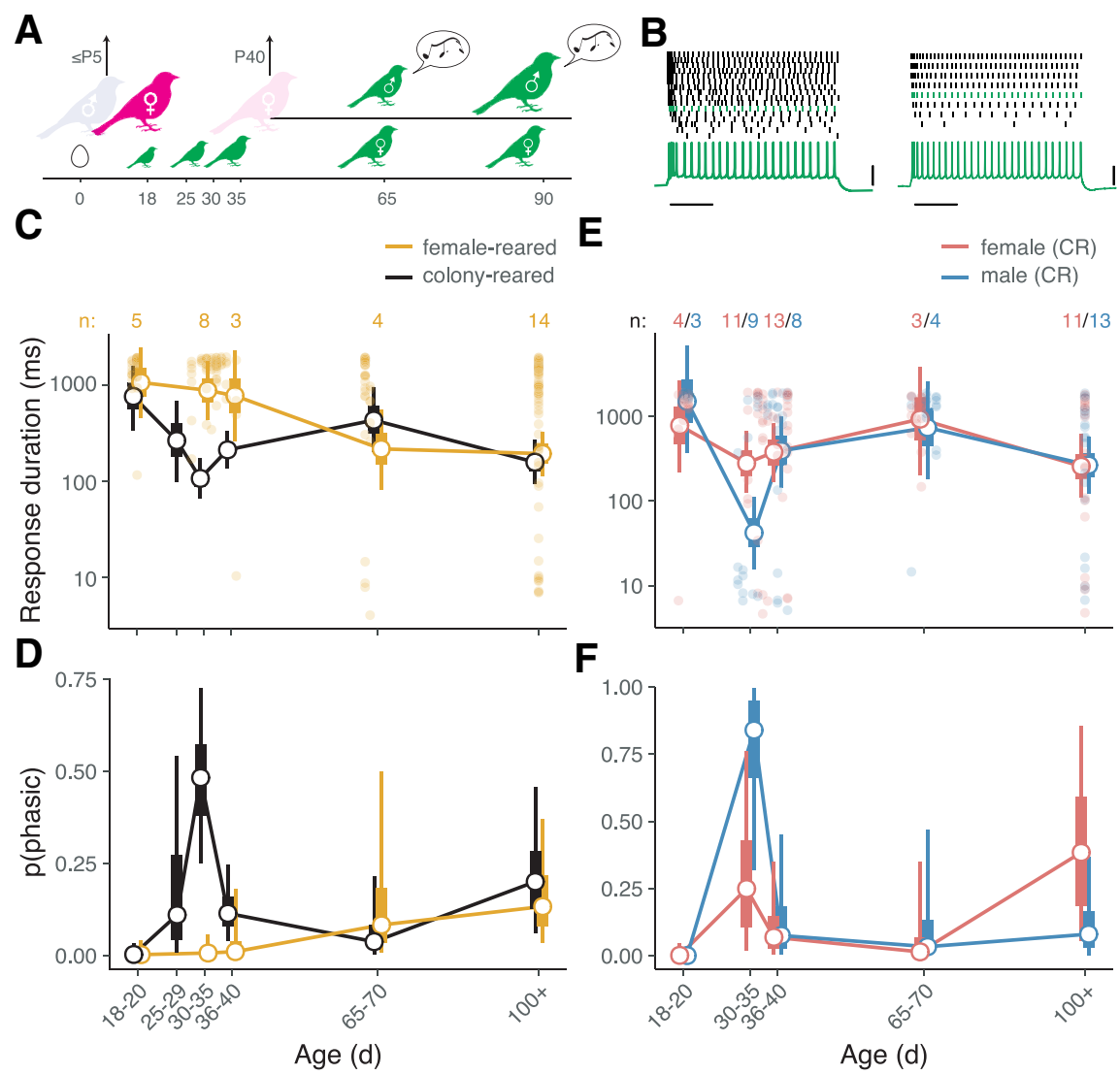

Figure 2. Phasic firing during the sensory learning period depends on sex and experience. $A$, Diagram of FR paradigm. The family is placed in acoustic isolation and the father removed $1-5 \mathrm{~d}$ after the first egg hatches. The mother is removed $\sim P 40$ and the male and female chicks are separated. Male chicks raised in this condition produce abnormal (isolate) song. $\boldsymbol{B}$, Examples of responses to step currents obtained from FR chicks (P20 and P32), as shown in Figure 1, C and D. Scale bars, $500 \mathrm{~ms}$ and $40 \mathrm{mV}$. C, Average response duration as a function of age in FR (yellow) and CR (black) animals. The format of the plot is as in Figure 1E. For visual clarity, data points for individual neurons are only shown for female-reared birds. Numerals indicate the number of FR birds. Between P30 - P35, response duration is lower (more phasic) in (R versus FR birds $(p<0.001)$. Among FR birds, average response duration at P18-P20 is greater (less phasic) than at P65-P70 $(p=0.04)$ and in adults $(p=0.002)$. D, Estimated proportion of phasic neurons. Phasic neurons are more prevalent in CR versus FR animals at P30 - P35 ( $p<0.001)$, and among FR animals, more prevalent in adults than at P18-P20 $(p=0.026)$. $E$, Average response duration as a function of age and sex in $C R$ animals. Responses are more phasic in males compared with females between P30-P35 $(p=0.004) . \boldsymbol{F}$, Proportion of phasic neurons as a function of age and sex. The apparent difference between males and females at P30 $-\mathrm{P} 35$ is not statistically significant $(p=0.16)$.

encoding a specific memory, or indirectly by facilitating auditory discrimination. To test this idea, we tracked changes in intrinsic firing patterns among CM neurons over time in birds that were not exposed to song (Fig. 2A). In contrast to CR controls, phasic firing was essentially absent in these FR animals during sensory acquisition (Fig. $2 B-D$ ), though it appeared in older animals (P65-70 and adults). Thus, intrinsic dynamics are affected by experience, but primarily during the time of song memorization.

The presence of sex-related differences in intrinsic excitability would also point to a relationship between intrinsic plasticity and song memorization, as only male zebra finches sing. To test this idea, we compared male and female CR birds, using plumage or genotyping to determine sex. As predicted, intrinsic dynamics were more phasic in males compared with females aged P30-P35, i.e., when plasticity is experience-dependent and phasic firing is most prevalent (Fig. 2E,F). The difference between males and females was statistically significant for response duration but not for the proportion of phasic neurons (Fig. 2E), indicating that the effect of sex is weaker than that of experience during P30-P35. In adults, there was no difference in phasic excitability between males and females.

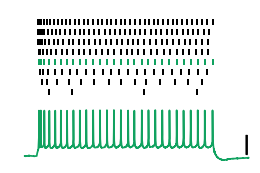

We also compared older male and female FR birds to determine whether the later phase of intrinsic plasticity depended on experience. Male FR birds go through sensorimotor vocal development despite the absence of a model to copy (Konishi, 1965; Volman and Khanna, 1995). Their developing songs are not species-typical but could nonetheless be a source of auditory experience for any birds that could hear them. We took this into account in our experimental design by separating all male and female FR chicks $\sim$ P40, thereby minimizing the exposure of young females to the attempts of their male siblings to sing. There was a trend toward more phasic excitability among CM neurons in adult FR males compared with adult FR females, but it was not statistically significant [GLMM: $\beta=-0.413$, CI: $(-0.89,0.10), p=0.096 ; n=12$ birds]. These data suggest that phasic excitability in adults is largely independent of experience hearing song.

\section{Intrinsic plasticity during song memorization requires exposure to colony noise}

We next sought to identify the specific acoustic factors required for intrinsic plasticity during the early, experiencedependent period. Although FR birds heard the calls of their mother and siblings, they were not exposed to normal adult song, which is exclusively produced by males in this species. The isolation boxes also created a much quieter, less complex acoustic environment for the FR birds. In contrast, CR chicks lived in individual cages with their father, mother, and siblings, and the cages were situated in a room containing $5-15$ other pairs and families. These conditions mimic the rich acoustic environment that these highly social birds would experience in the wild. Colony noise comprises songs and several different kinds of calls and is characterized by large amplitude modulations and a complex spectrotemporal modulation spectrum (Fig. 3).

To dissociate the effects of specific types of early auditory exposure, i.e., tutor song versus colony noise, we examined two additional rearing conditions (Fig. 4A). Like FR birds, PR chicks were housed in acoustic isolation boxes, but the father was not removed. Thus, PR chicks heard song in a quiet environment and received the same level of parental care and social interaction as CR chicks. VI birds were housed in the colony room, but in the absence of the father and with the addition of opaque barriers between cages. Thus, VI chicks heard colony noise, but could not socially interact with any adult males. This lack of social interaction appears to be critical for song acquisition, as despite hearing song, VI chicks produce abnormal adult songs that resemble those of FR birds (Morrison and Nottebohm, 1993). After VI and PR chicks reached 30-40 d of age, we recorded responses to step currents in CM slices. Surprisingly, the effect of exposing birds to a tutor (the PR condition) was small and not statistically signifi- 
cant, whereas exposing birds to colony noise in the absence of a tutor (the VI condition) partially rescued phasic excitability. The effect of VI rearing nearly reached statistical significance (Fig. $4 B, C$ ), despite the small sample size ( $n=4$ birds). This result suggests that a complex acoustic environment, rather than song exposure, is necessary for phasic firing to emerge.

\section{Exposure to colony noise increases expression of a low-threshold potassium channel}

To confirm the key effects of experience on intrinsic excitability, we tested for correlated changes in the expression of Kv1.1, which we hypothesized to be mediating phasic firing. In a previous study, pharmacological experiments in juveniles (P34-P40) showed that whereas firing patterns were unaffected by blocking fast synaptic transmission, phasic neurons became more tonic when 4 -AP or $\alpha$-dendrotoxin was applied, implicating a channel in the Kv1.x family (Chen and Meliza, 2018). The zebra finch expression atlas (ZEBrA Database, 2019) shows expression in CM of two genes in this family, KCNA1 and KCNA6, which correspond to Kv1.1 and Kv1.6, respectively. We searched for antibodies to stain these channels and found a monoclonal antibody against the C-terminus of rat Kv1.1 that produced staining consistent with the RNA expression patterns in the atlas.

Using this antibody, we quantified the proportion of neurons expressing Kv1.1 to test three predictions: first, expression should increase between P18-20 and P30-P35; second, expression should be greater in CR birds at P30-P35 than in age-matched FR birds; and third, expression in VI birds at P30-P35 should also be greater than in age-matched FR animals. All of these predictions were confirmed. Kv1.1 was highly expressed in CM neurons during P30-P35 (Fig. 5A). The proportion of neurons expressing Kv1.1 was higher in P30-P35 CR birds compared with agematched FR animals and younger CR birds (Fig. $5 B$ ). In VI birds, there were also more Kv1.1-expressing neurons than in agematched FR animals, though somewhat less than in CR birds, consistent with our electrophysiological results (Fig. 4B,C).

In all rearing conditions, staining appeared punctate and was located in the cell body outside the nucleus, or sometimes along what looked like processes (Fig. 5C). Kv1.1-positive neurons were consistently more prevalent in medial compared with lateral sections (odds ratio $=1.20 ; p=0.02$ ). This difference could be related to the functional and hodological differences between CLM and CMM (Jeanne et al., 2011); however, given the small size of this effect and the small proportion of neurons we recorded from CMM, the present study does not have sufficient power to determine whether intrinsic dynamics vary between these anatomically amorphous regions. There was no difference between sexes $(p=0.68)$ or between hemispheres $(p=0.88)$. There is no well validated membrane marker in zebra finches, so we were unable to determine what proportion of the signal was colocalized with the plasma membrane.

\section{Discussion}

Our results demonstrate that intrinsic neuronal dynamics in CM are highly plastic during development. Between fledging and

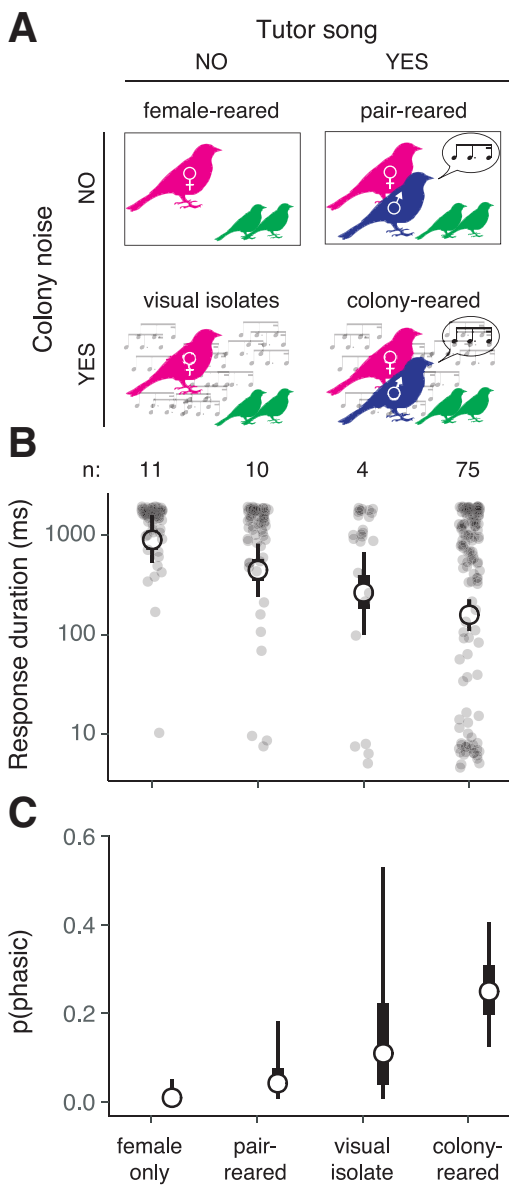

Figure 4. Phasic dynamics are partially rescued by exposure to a complex acoustic environment. $\boldsymbol{A}$, Schematic of rearing conditions. $\boldsymbol{B}$, Average response duration in P30-P40 birds for each condition. Format is as in Figure $1 E$. Compared with FR birds, average response duration is lower (more phasic) in CR birds (GLMM: $p<0.001$ ) and nearly significantly lower in VI birds $(p=0.052)$. C, Proportion of phasic neurons in each condition. Compared with FR birds, there are more phasic neurons in CR birds $(p=0.006)$. 
A
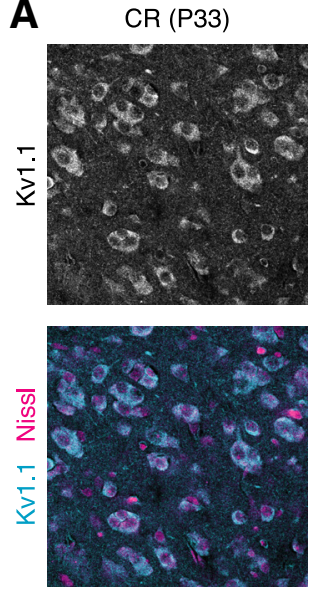

FR (P30)
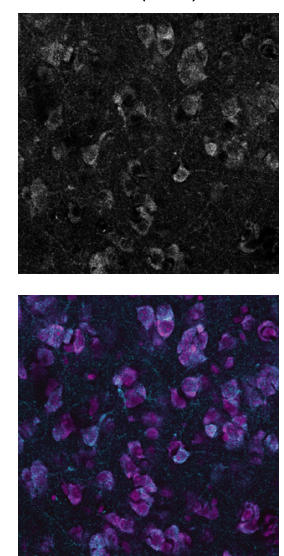
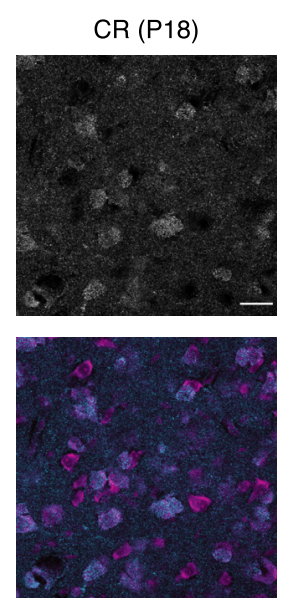

B

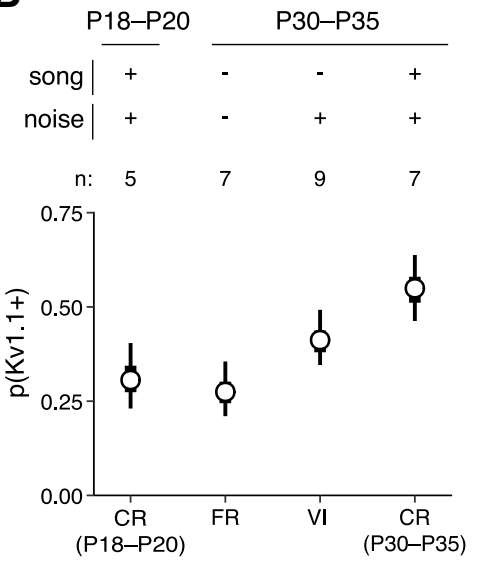

C
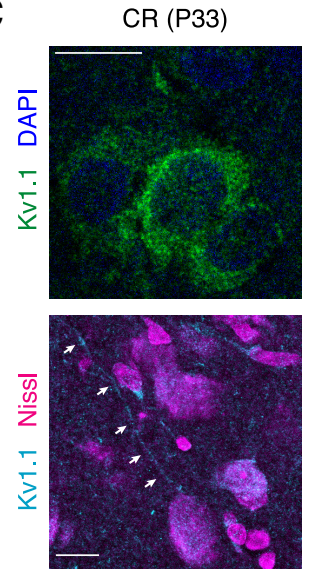

Figure 5. Kv1.1 expression depends on age and experience. $\boldsymbol{A}$, Examples of $\alpha$-Kv1.1 staining in different rearing conditions. Images are single confocal slices ( $0.44 \mathrm{~m}$ ) with identical laser, gain, and contrast settings for Kv1.1. Columns correspond to samples from different ages and rearing conditions. Top row, Kv1.1 signal in grayscale; bottom row, composite of Kv1.1 (cyan) with a fluorescent Nissl stain (magenta). Scale bar, $20 \mu \mathrm{m}$. B, Estimated proportion of Kv1.1-positive neurons at each age/condition tested. Compared with FR birds at P30-P35, the proportion of Kv1.1-positive neurons is higher in age-matched CR birds (GLMM: $p=0.001$ ) and VI birds ( $p=0.03$ ). The proportion of Kv1.1-positive neurons is also greater in CR birds at P30 -P35 than in CR birds at P18-P20 ( $p=0.004)$. C, Higher-power images $(63 \times)$ of Kv1.1 staining in cell bodies (top) and processes (bottom). Scale bars, $10 \mu \mathrm{m}$.

adulthood, there are three shifts in the distribution of intrinsic dynamics, and the timing of these changes coincides with key milestones in the development of vocal communication in this species. In the youngest animals we examined (P18-P20), the overwhelming majority of broad-spiking neurons exhibited sustained, regular spiking patterns typical of excitatory cortical neurons. At the age when young males begin to memorize a song to copy (P30-P35), $\sim 50 \%$ of the cells had shifted to only producing transient responses. Within several days (P36-P40), intrinsic dynamics then shifted back to tonic firing. By the age when males start intensively practicing song production (P65-P70), only $\sim 10 \%$ of the CM neurons were phasic. Phasic excitability again increased in adults, i.e., at ages when males have finalized the song they will produce for the rest of their lives, and when both sexes are listening to song in social interactions (including, but not limited to mate selection). Each of these phases of plasticity depend on experience and sex to differing degrees.

The earliest phase of intrinsic plasticity occurs at the beginning of the song memorization period and depends on experience and sex. We found that phasic excitability fails to emerge during this period in FR birds and is stronger in males than in females. These results are consistent with a role in song memorization, but not in directly encoding a memory of the tutor song. Phasic excitability is transient, disappearing almost entirely during the sensorimotor learning phase, so any engrams that depended on this aspect of intrinsic excitability would presumably be lost. More importantly, phasic excitability does not depend on exposure to a tutor. Birds raised with a tutor in quiet conditions (i.e., pair-reared) show little phasic excitability between P30-P35. In contrast, chicks raised without visual or social access to a tutor but in the noisy and acoustically complex environment of the colony (i.e., visual isolates) show partial rescue of phasic excitability. Thus, phasic excitability is a consequence of the acoustic (and possibly social) environment rather than the memorization of a single tutor song.

A number of previous studies in songbirds have examined the effects of acoustic experience on auditory responses (Adret et al., 2012; Amin et al., 2013; Moore and Woolley, 2019), perception (Sturdy et al., 2001; Chen et al., 2017), and intrinsic excitability (Ross et al., 2019). However, the assumption has been that the acoustic structure of the tutor song is the most important factor shaping development, and the effects of the acoustic background have not been considered. More generally, juveniles are typically prevented from hearing a tutor by placing them in acoustic isolation boxes without any adult males present, but this also eliminates the complex auditory scene of songs and calls found in breeding colonies, which typically comprise many individuals (Olson et al., 2014; McCowan et al., 2016). The present study addresses this confound by separately controlling social exposure to a tutor, which is necessary for song memorization, and exposure to colony noise, which includes song but is not sufficient for normal vocal development (Morrison and Nottebohm, 1993). By dissociating these factors, our results clearly support the hypothesis that experience-dependent plasticity in the songbird auditory system can take place independently of song memorization.

Birds raised with a tutor in quiet environments have not been shown to exhibit any overt deficits in song learning (Tchernichovski and Nottebohm, 1998) or perception, so the behavioral consequences of an impoverished acoustic environment, and the reduction in phasic firing that results, are not known. However, a parsimonious interpretation of the present results is that intrinsic plasticity in CM is an adaptation to noise. As vocal learners raised in social environments, zebra finch chicks are faced with a challenging version of the cocktail-party problem: not only do they have to separate the song of a single tutor from a background with nearly identical spectrotemporal statistics, they presumably are still learning the structure and nuances of their species' song. The phasic dynamics induced by exposure to a colony environment may be critical to solving this problem, especially in younger animals in whom inhibitory circuitry may still be developing (Schneider and Woolley, 2013; Takesian and Hensch, 2013; Vallentin et al., 2016). In subcortical auditory areas, low-threshold potassium currents facilitate detecting brief periods of coherent excitation within a noisy signal (Golding et al., 1995; Svirskis et al., 2002), an effect that we also observed in a recent computational modeling study of CM (Bjoring and Meliza, 2019). If this hypothesis is the case, PR birds may therefore fail to develop phasic firing because they do not need it to memorize a song in a quiet environment.

The dramatic change in physiological properties that occurs during song memorization is mirrored by increased expression of Kv1.1, a low-threshold potassium channel that facilitates phasic 
firing (Bal and Oertel, 2001). Among CR birds aged P30-P35, there was close quantitative agreement between the proportion of neurons expressing Kv1.1 and the proportion of neurons that only exhibited phasic firing. However, in FR and younger CR animals, Kv1.1 was expressed in close to $30 \%$ of cells, but there were essentially no electrophysiologically phasic neurons in either of these groups. This discrepancy likely stems from the highly nonlinear relationship between low-threshold potassium conductance and phasic firing (Rothman and Manis, 2003; Chen and Meliza, 2018), and from the fact that much of the Kv1.1 staining was within the cell body (Fig. 5C), suggesting that most of the channels are not at the cellular membrane where they need to be to influence voltage dynamics. Indeed, Kv1.1 has a strong ER retention signal (Manganas et al., 2001), so expression is likely necessary, but not sufficient for phasic excitability (Akter et al., 2018).

Intrinsic dynamics continue to change throughout development. After peaking between P30-P35, phasic excitability decreases almost to the P18-P20 baseline by $\mathrm{P} 65$, which is the age when the sensory acquisition phase ends, and birds are no longer able to learn new song elements (Gobes et al., 2019). Phasic excitability again increases as birds become adults, but in contrast to the early phase, this change does not appear to depend on experience or sex, as there were no statistically significant differences in adults between FR and CR animals, or between males and females. The developmental profile of phasic excitability (Fig. $2 C, D)$ may therefore reflect two distinct mechanisms: one early, transient, experience- and sex-dependent; the other late, longlasting, and independent of experience or sex. Indeed, it is important to note that the pharmacological data from our earlier study (Chen and Meliza, 2018) and the channel expression data from the present study (Fig. 5), which implicate Kv1.1 in phasic firing, are only from juvenile birds, so it is possible that an entirely different electrophysiological mechanism is responsible for phasic excitability in adults, for example $B_{K}$-type, $S_{K}$-type, or leak potassium currents (Sivaramakrishnan and Oliver, 2001; Daou et al., 2013; Dagostin et al., 2015).

At this time, we can only speculate as to the function of the later phases of intrinsic plasticity. If, as we posit, phasic excitability facilitates auditory processing in noisy conditions, then it would clearly have a role to play in adults. Both male and female zebra finches need to hear song amid colony noise to communicate, and thus share similar requirements for robust and sensitive auditory perception. This could explain why the late phase of plasticity does not depend on sex, but it does not shed much light on why phasic excitability would decrease in older juveniles or why there is no effect of experience on intrinsic excitability in adults. Another possibility, suggested by a recent study of intrinsic plasticity in the premotor nucleus HVC, is that changes in intrinsic dynamics may also be gating synaptic plasticity. In HVC projection neurons, exposure to a tutor song appears to transiently suppress specific currents during the song memorization period, including a putative $I_{\mathrm{h}}$ current (Ross et al., 2017, 2019), which has been implicated in the suppression of long-term potentiation (Nolan et al., 2004). On this view, the periods of increased phasic excitability in CM could reflect intervals of heightened plasticity, with an important distinction between CM and $\mathrm{HVC}$ being that CM is known to be a site of learning-related functional plasticity in adulthood (Gentner and Margoliash, 2003). Although this interpretation is difficult to reconcile with the data from PR animals, who lack phasic firing between P30P35 but can still develop normal song, future work should consider how changes in intrinsic properties shape the capacity of $\mathrm{CM}$ for learning.
Experience-dependent plasticity in sensory systems is common during development (Sanes and Woolley, 2011; Levelt and Hübener, 2012). In rodent auditory cortex, manipulating experience during an early critical period has long-lasting effects on functional response properties (Zhang et al., 2001; de VillersSidani et al., 2007), perception (Han et al., 2007), synaptic connections (Dorrn et al., 2010; Sun et al., 2010), and intrinsic properties (Kotak et al., 2005). The early, experience-dependent plasticity we observed here is consistent with these findings and with the general consensus that neural circuits adapt during development to the structure of sensory input. Indeed, whereas many previous studies required deafening or thousands of repetitions of artificial stimuli to induce plasticity, we found that placing birds in a more natural social environment was sufficient to cause large changes in channel expression and physiology. This environment is characterized by noise only $15 \mathrm{~dB}$ louder than the isolated conditions, but with a much more complex acoustic structure, which may be the primary factor driving plasticity (Bao et al., 2013). The rich social environment experienced by VI and CR chicks, with opportunities for vocal interactions against a complex noise background, may also have contributed. Our results therefore provide a new level of support for the biological significance of developmental plasticity to auditory perception and vocal communication.

Although phasic spiking appears to be rare in mammalian auditory cortex (Metherate and Aramakis, 1999), Kv1.1 is widely expressed in cortical neurons (Higgs and Spain, 2011; Dehorter et al., 2015), so experience-dependent intrinsic plasticity may also have implications for acoustic communication in a broad range of species. Indeed, any species that uses acoustic signaling in large social groups requires an auditory system that can decode these signals against a background with nearly identical spectrotemporal statistics. Understanding how neural circuits adapt to this kind of interference may yield insight into this challenging signalprocessing problem. Intrinsic plasticity may also be of particular relevance to human speech, as a number of genes implicated as risk factors for language-learning disorders, including CNTNAP2 and KIAA0319, are involved in Kv1.1 trafficking (Strauss et al., 2006) and in regulation of intrinsic excitability (Centanni et al., 2014). Thus, we recognize that the mechanism implicated in our data may be relevant to future studies of the pathogenesis and therapeutic interventions related to these conditions.

\section{References}

Adret P, Meliza CD, Margoliash D (2012) Song tutoring in presinging zebra finch juveniles biases a small population of higher-order song-selective neurons toward the tutor song. J Neurophysiol 108:1977-1987.

Akter N, Adachi R, Kato A, Fukaya R, Kuba H (2018) Auditory input shapes tonotopic differentiation of Kv1.1 expression in avian cochlear nucleus during late development. J Neurosci 38:2967-2980.

Amin N, Gastpar M, Theunissen FE (2013) Selective and efficient neural coding of communication signals depends on early acoustic and social environment. PLoS One 8:e61417.

Bal R, Oertel D (2001) Potassium currents in octopus cells of the mammalian cochlear nucleus. J Neurophysiol 86:2299-2311.

Bao S, Chang EF, Teng CL, Heiser MA, Merzenich MM (2013) Emergent categorical representation of natural, complex sounds resulting from the early post-natal sound environment. Neuroscience 248:30-42.

Bjoring MC, Meliza CD (2019) A low-threshold potassium current enhances sparseness and reliability in a model of avian auditory cortex. PLoS Comput Biol 15:e1006723.

Bottjer SW (2005) Silent synapses in a thalamo-cortical circuit necessary for song learning in zebra finches. J Neurophysiol 94:3698-3707.

Centanni TM, Booker AB, Sloan AM, Chen F, Maher BJ, Carraway RS, Khodaparast N, Rennaker R, LoTurco JJ, Kilgard MP (2014) Knockdown of the dyslexia-associated gene Kiaa0319 impairs temporal responses to 
speech stimuli in rat primary auditory cortex. Cereb Cortex 24:1753-1766.

Chen AN, Meliza CD (2018) Phasic and tonic cell types in the zebra finch auditory caudal mesopallium. J Neurophysiol 119:1127-1139.

Chen Y, Clark O, Woolley SC (2017) Courtship song preferences in female zebra finches are shaped by developmental auditory experience. Proc Biol Sci 284:20170054.

Dagostin AA, Lovell PV, Hilscher MM, Mello CV, Leão RM (2015) Control of phasic firing by a background leak current in avian forebrain auditory neurons. Front Cell Neurosci 9:471.

Daou A, Ross MT, Johnson F, Hyson RL, Bertram R (2013) Electrophysiological characterization and computational models of HVC neurons in the zebra finch. J Neurophysiol 110:1227-1245.

Dehorter N, Ciceri G, Bartolini G, Lim L, del Pino I, Marín O (2015) Tuning of fast-spiking interneuron properties by an activity-dependent transcriptional switch. Science 349:1216-1220.

de Villers-Sidani E, Chang EF, Bao S, Merzenich MM (2007) Critical period window for spectral tuning defined in the primary auditory cortex (A1) in the rat. J Neurosci 27:180-189.

Dorrn AL, Yuan K, Barker AJ, Schreiner CE, Froemke RC (2010) Developmental sensory experience balances cortical excitation and inhibition. Nature 465:932-936.

Doupe AJ, Kuhl PK (1999) Birdsong and human speech: common themes and mechanisms. Annu Rev Neurosci 22:567-631.

Eales LA (1985) Song learning in zebra finches: some effects of song model availability on what is learnt and when. Anim Behav 33:1293-1300.

Elie JE, Theunissen FE (2016) The vocal repertoire of the domesticated zebra finch: a data-driven approach to decipher the information-bearing acoustic features of communication signals. Anim Cogn 19:285-315.

Gentner TQ, Margoliash D (2003) Neuronal populations and single cells representing learned auditory objects. Nature 424:669-674.

Gobes SMH, Jennings RB, Maeda RK (2019) The sensitive period for auditory-vocal learning in the zebra finch: consequences of limitedmodel availability and multiple-tutor paradigms on song imitation. Behav Processes 163:5-12.

Golding NL, Robertson D, Oertel D (1995) Recordings from slices indicate that octopus cells of the cochlear nucleus detect coincident firing of auditory nerve fibers with temporal precision. J Neurosci 15:3138-3153.

Han YK, Köver H, Insanally MN, Semerdjian JH, Bao S (2007) Early experience impairs perceptual discrimination. Nat Neurosci 10:1191-1197.

Higgs MH, Spain WJ (2011) Kvl channels control spike threshold dynamics and spike timing in cortical pyramidal neurones. J Physiol 589:5125-5142.

Jeanne JM, Thompson JV, Sharpee TO, Gentner TQ (2011) Emergence of learned categorical representations within an auditory forebrain circuit. J Neurosci 31:2595-2606.

Konishi M (1965) The role of auditory feedback in the control of vocalization in the white-crowned sparrow. Z Tierpsychol 22:770-783.

Kotak VC, Fujisawa S, Lee FA, Karthikeyan O, Aoki C, Sanes DH (2005) Hearing loss raises excitability in the auditory cortex. J Neurosci 25:3908-3918.

Kuhl PK (2004) Early language acquisition: cracking the speech code. Nat Rev Neurosci 5:831-843.

Levelt CN, Hübener M (2012) Critical-period plasticity in the visual cortex. Annu Rev Neurosci 35:309-330.

Manganas LN, Wang Q, Scannevin RH, Antonucci DE, Rhodes KJ, Trimmer JS (2001) Identification of a trafficking determinant localized to the Kv1 potassium channel pore. Proc Natl Acad Sci U S A 98:14055-14059.

Marler P, Tamura M (1964) Culturally transmitted patterns of vocal behavior in sparrows. Science 146:1483-1486.

Maye J, Werker JF, Gerken L (2002) Infant sensitivity to distributional information can affect phonetic discrimination. Cognition 82:B101-B111.

McCowan LSC, Mariette MM, Griffith SC (2016) The size and composition of social groups in the wild zebra finch. Emu 115:191-198.

Meliza CD, Margoliash D (2012) Emergence of selectivity and tolerance in the avian auditory cortex. J Neurosci 32:15158-15168.

Metherate R, Aramakis VB (1999) Intrinsic electrophysiology of neurons in thalamorecipient layers of developing rat auditory cortex. Brain Res Dev Brain Res 115:131-144.

Moore JM, Woolley SMN (2019) Emergent tuning for learned vocalizations in auditory cortex. Nat Neurosci 22:1469-1476.
Morrison RG, Nottebohm F (1993) Role of a telencephalic nucleus in the delayed song learning of socially isolated zebra finches. J Neurobiol 24:1045-1064.

Nolan MF, Malleret G, Dudman JT, Buhl DL, Santoro B, Gibbs E, Vronskaya S, Buzsáki G, Siegelbaum SA, Kandel ER, Morozov A (2004) A behavioral role for dendritic integration: HCN1 channels constrain spatial memory and plasticity at inputs to distal dendrites of CA1 pyramidal neurons. Cell 119:719-732.

Olson CR, Wirthlin M, Lovell PV, Mello CV (2014) Proper care, husbandry, and breeding guidelines for the zebra finch, Taeniopygia guttata. Cold Spring Harb Protoc 2014:1243-1248.

Ross MT, Flores D, Bertram R, Johnson F, Hyson RL (2017) Neuronal intrinsic physiology changes during development of a learned behavior. eNeuro 4:ENEURO.0297-17.2017.

Ross MT, Flores D, Bertram R, Johnson F, Wu W, Hyson RL (2019) Experience-dependent intrinsic plasticity during auditory learning. J Neurosci 39:1206-1221.

Rothman JS, Manis PB (2003) The roles potassium currents play in regulating the electrical activity of ventral cochlear nucleus neurons. J Neurophysiol 89:3097-3113.

Sanes DH, Woolley SM (2011) A behavioral framework to guide research on central auditory development and plasticity. Neuron 72:912-929.

Schneider DM, Woolley SM (2013) Sparse and background-invariant coding of vocalizations in auditory scenes. Neuron 79:141-152.

Singh NC, Theunissen FE (2003) Modulation spectra of natural sounds and ethological theories of auditory processing. J Acoust Soc Am 114:3394-3411

Sivaramakrishnan S, Oliver DL (2001) Distinct K currents result in physiologically distinct cell types in the inferior colliculus of the rat. J Neurosci 21:2861-2877.

Strauss KA, Puffenberger EG, Huentelman MJ, Gottlieb S, Dobrin SE, Parod JM, Stephan DA, Morton DH (2006) Recessive symptomatic focal epilepsy and mutant contactin-associated protein-like 2. N Engl J Med 354:1370-1377.

Sturdy CB, Phillmore LS, Sartor JJ, Weisman RG (2001) Reduced social contact causes auditory perceptual deficits in zebra finches, Taeniopygia guttata. Anim Behav 62:1207-1218.

Sun YJ, Wu GK, Liu BH, Li P, Zhou M, Xiao Z, Tao HW, Zhang LI (2010) Fine-tuning of pre-balanced excitation and inhibition during auditory cortical development. Nature 465:927-931.

Svirskis G, Kotak V, Sanes DH, Rinzel J (2002) Enhancement of signal-tonoise ratio and phase locking for small inputs by a low-threshold outward current in auditory neurons. J Neurosci 22:11019-11025.

Takesian AE, Hensch TK (2013) Balancing plasticity/stability across brain development. Prog Brain Res 207:3-34.

Tchernichovski O, Nottebohm F (1998) Social inhibition of song imitation among sibling male zebra finches. Proc Natl Acad Sci U S A 95:8951-8956.

Vallentin D, Kosche G, Lipkind D, Long MA (2016) Inhibition protects acquired song segments during vocal learning in zebra finches. Science 351:267-271.

Volman SF, Khanna H (1995) Convergence of untutored song in groupreared zebra finches (Taeniopygia guttata). J Comp Psychol 109:211-221.

Wang X, Lu T, Bendor D, Bartlett E (2008) Neural coding of temporal information in auditory thalamus and cortex. Neuroscience 157:484-494.

Wang Y, Brzozowska-Prechtl A, Karten HJ (2010) Laminar and columnar auditory cortex in avian brain. Proc Natl Acad Sci U S A 107:12676-12681.

Werker JF, Lalonde CE (1988) Cross-language speech perception: initial capabilities and developmental change. Dev Psychol 24:672-683.

Woolley SM (2012) Early experience shapes vocal neural coding and perception in songbirds. Dev Psychobiol 54:612-631.

ZEBrA Database (2019) Oregon Health and Science University, Portland, OR. Available at http://www.zebrafinchatlas.org.

Zhang LI, Bao S, Merzenich MM (2001) Persistent and specific influences of early acoustic environments on primary auditory cortex. Nat Neurosci 4:1123-1130.

Zhao S, Ting JT, Atallah HE, Qiu L, Tan J, Gloss B, Augustine GJ, Deisseroth K, Luo M, Graybiel AM, Feng G (2011) Cell type-specific channelrhodopsin-2 transgenic mice for optogenetic dissection of neural circuitry function. Nat Methods 8:745-752. 